\title{
Competition-Based Cell Assay Employing Soluble T Cell Receptors to Assess MHC Class II Antigen Processing and Presentation
}

\author{
Carley Tasker, ${ }^{1}$ Jenny Patel, ${ }^{1}$ Vibha Jawa, ${ }^{1}$ and Jad Maamary ${ }^{1,2}$
}

Received 13 October 2020; accepted 22 December 2020; published online 18 January 2021

Abstract. Accurate assessment of antigen-specific immune responses is critical in the
development of safe and efficacious biotherapeutics and vaccines. Endosomal processing of a
protein antigen followed by presentation on major histocompatibility complex (MHC) class
II constitute necessary steps in the induction of CD4+ T cell immune responses. Current
preclinical methods for assessing immunogenicity risk consist of in vitro cell-based assays and
computational prediction tools. Cell-based assays are time and labor-intensive while in silico
methodologies have limitations. Here, we propose a novel cell-based assay capable of
investigating an antigen's endosomal processing and MHC class II presentation capabilities.
This novel assay relies on competition between epitopes for MHC class II binding and
employs labeled soluble T cell receptors (sTCRs) as detectors of epitope presentation.

KEY WORDS: antigen presentation; APC; HLA-DR; MHC; TCR.

\section{INTRODUCTION}

Development of antigen-specific immunological memory is critical in establishing long-lived protection against infections. Immunological memory, including the generation of high affinity matured antibodies, is a multi-step process dependent on antigen-specific T cell help (1). To initiate this process, antigen presenting cells (APCs) uptake and digest protein antigen into linear epitopes. These epitopes are loaded on to major histocompatibility complex (MHC) class II and presented to $\mathrm{T}$ cell receptors (TCRs) (2). TCR recognition by antigen-specific CD4+ helper $\mathrm{T}(\mathrm{Th})$ cells leads to cellular activation and expansion $(1,3)$. Activated Th cells provide help to antigen-specific B cells resulting in the generation of antibody secreting plasma cells and long-lived memory B cells $(1,4)$.

Antibody-specific immunity is critical in combating infection and developing efficacious vaccines. In unwanted cases, antigen-specific immunity can develop in response to protein therapeutics leading to the generation of antidrug antibodies (ADA) that can impact both drug efficacy and tolerability $(5,6)$. To assess the likelihood of generating an antigen-specific immune response, several analytical tools that employ primary human immune cells have been developed. Dendritic cell (DC)-T cell assays measure $\mathrm{CD} 4+\mathrm{T}$ cell proliferation in response to antigen

\footnotetext{
${ }^{1}$ Predictive and Clinical Immunogenicity, Merck \& Co., Inc., Kenilworth, New Jersey, USA.

${ }^{2}$ To whom correspondence should be addressed. (e-mail: jad.maamary@merck.com)
}

pulsed matured autologous DCs and peripheral blood mononuclear cell (PBMC) assays quantify cytokine response and $\mathrm{CD} 4+\mathrm{T}$ cell activation in antigen pulsed heterogenous cell cultures. These assays can detect antigen-specific $\mathrm{CD} 4+\mathrm{T}$ cell responses in naïve donors which may correlate with ef drug immunogenicity. For robustness and wide HLA coverage, multiple human blood donors are needed for analysis, making these assays both labor intensive and time consuming (7, 8). Additional limitations are the inability of these assays to easily distinguish immunogenic epitopes within a full protein.

To specifically determine epitopes presented on MHC, an LC-MS/MS approach referred as MHC-associated peptide proteomics (MAPPs) assay is utilized. In this approach, APCs are pulsed with test antigen, and MHC II-associated peptides are immunoprecipitated. Peptides subsequently eluted from MHC II molecules are then sequenced by LC-MS/MS $(9,10)$. While MAPPs can accurately identify epitopes in the context of a full protein, sensitivity varies depending on the protocol (11). Use of primary human cells requires the inclusion of many donors for diverse HLA coverage and further complicates outcomes. These assays also require high number cells which impose practical limits on number conditions analyzed for each compound (9).

To address the inability to test large numbers of candidates/compounds in cell-based assays, high-throughput in silico algorithms are utilized. These predictive methods are trained on large sets of MHC class II binding data and can specifically target areas of high immunogenicity risk within the protein sequence (12). Despite the evolving robustness of in silico methods, many of these tools are unable to predict cellular processing and trafficking of peptides especially for 
MHC-II epitopes. Integration of MAPPs data and antigen cleavage data has improved the accuracy of these tools (1315). However, predictions are limited by the breadth and accuracy of data sources used for training these tools.

Here we propose the development of a novel cellbased assay that takes advantage of the competition between epitopes to bind MHC class II and employs labeled soluble $\mathrm{T}$ cell receptors (sTCRs) to detect peptideMHC complexes (pMHC) $(16,17)$. Soluble TCRs have been proposed as a novel immunotherapy engineered for targeted delivery of oncology drugs and immunomodulatory agents $(18,19)$. However, this is the first example where sTCRs are employed to assess antigen presentation. To overcome limitations of MHC-II heterogenicity in primary cell-based assays, B lymphoblastoid cells (BLCL) mono-allelic for the HLA-DRB1 locus are employed as APCs. This assay will determine MHC class II presentation based on the concept that epitopes restricted by the same MHC molecule compete for presentation (16). Mono-allelic APCs will be pulsed with a reference epitope alone or in the presence of a test compound. Labeled sTCRs recognizing the reference epitope/MHC complex ( $\mathrm{e}_{\mathrm{r}} \mathrm{MHC}$ ) will be used to detect reference epitope presentation on MHC class II. Decreased sTCR/e $\mathrm{e}_{\mathrm{r}} \mathrm{MHC}$ binding signal in the presence of a test compound will indicate test compound presentation on MHC class II. We demonstrate that assay-detected epitopes from test compounds correlated with in silico predictions and standard MAPPs assay analysis. The assay proposed here has diverse applications ranging from vaccine development, neoantigen evaluation, and immunogenicity analysis and de-risking.

\section{MATERIALS AND METHODS}

\section{Soluble T Cell Receptor Expression and Purification}

Expression and purification of soluble $\mathrm{T}$ cell receptors were performed by GenScript Biotech (Piscataway, NJ) using the following conditions.

Preparation of Influenza HA sTCR. Expi293F cells were transfected with pUC57 plasmid containing HA sTCR using ExpiFectamine $^{\mathrm{TM}} 293$ reagent and cultured in serum-free Expi293 ${ }^{\mathrm{TM}}$ Expression Medium (Thermo Fisher Scientific) at $37^{\circ} \mathrm{C}$ at $8 \% \mathrm{CO}_{2}$ on an orbital shaker. Cell culture supernatants were collected on day 6 for purification.

Preparation of Stable Cell Lines Expressing HIV gag p24 $s T C R$. CHO-K1SP cells were transfected with pUC57 plasmid containing p24 sTCR twice and expression was detected $48 \mathrm{~h}$ post-transfection by Western blot. Transfected cells maintained in selection medium (CD CHO medium, $25 \mu \mathrm{M}$ MSX, 200x anti-clumping agent) were divided into pools and screened by Dot-Blot and SDS-PAGE. Single clones from the top 12 cell pools were obtained by limiting dilution. The top 10 clones were maintained in Dynamis medium with 200x anti-clumping agent and incubated at $37^{\circ} \mathrm{C}, 5 \% \mathrm{CO}_{2}$. Feed $\mathrm{B}$ solution was added on day $4,6,8,10$, and 12 and glucose was added to maintain the concentration range from 4 to $6 \mathrm{~g} / \mathrm{L}$.
Supernatant from the top producing clone was harvested on the last day for SDS-PAGE analysis and purification.

Purification. Filtered cell culture media were loaded on to an affinity purification column and two-step purification was performed using Anti-DYKDDDDK G1 affinity resin (GenScript cat\# L00432) and HiLoad16/600 Superdex200pg. The eluted fractions were pooled and exchanged to the final formulation buffer (PBS, $\mathrm{pH}$ 7.2). The purified protein was analyzed by SDS-PAGE, Western blot, and SEC-HPLC analysis to determine molecular weight and purity. The concentration was determined by Bradford assay with a BSA standard (Thermo Fisher cat\# 23236). For all lots, purity of the sTCR was above $80 \%$ as measured by SEC-HPLC, and endotoxin level below 2.1EU/mg as measured by LAL Endotoxin Assay Kit (GenScript cat\# L00350).

\section{Synthesis of Omomyc Dimer}

The Omomyc protein sequence was assembled by solid phase synthesis on a Microwave Liberty Blue synthesizer (CEM, Matthews, NC). Released and deprotection of Omomyc from its solid support were achieved by stirring the peptidyl-resin in TFA (trifluoroacetic acid)/water/TIS (triisopropylsilane)/DTT (dithiothreitol) (93:3:3:1, v:v:v:w; $10 \mathrm{~mL} / \mathrm{g}$ of peptidylresin) for $2-3 \mathrm{~h}$ at room temperature. The crude protein product was precipitated and washed with diethyl ether. It was then purified to $>90 \%$ purity using preparative masstriggered HPLC on C18 Reverse Phase columns and linear gradients of acetonitrile in water, both buffered with $0.1 \%$ TFA. The HPLC fractions containing a pure protein product were pooled and lyophilized to yield Omomyc as a white solid in TFA salt form. Conversion of the TFA to acetate salt form of the protein was performed using a HiTrap Q HP strong anion exchange column (5 mL; GE Healthcare). Purified Omomyc monomer was dimerized via disulfide formation by stirring it in a solution of Water (48.0 mL) and 10X TBS ( $\mathrm{pH} 7.4)$ (48 mL), adjusted to $\mathrm{pH} 7.8$ by addition of $1.2 \mathrm{~mL}$ of $5 \mathrm{M}$ $\mathrm{NaOH}$, followed by addition of DMSO $(21.0 \mathrm{~mL})$. HPLC fractions containing a pure protein product were pooled and lyophilized to yield Omomyc dimer as a white solid in TFA salt form. Conversion of the TFA to acetate salt form of the protein was performed using a custom column packed with Fast Flow Q Sepharose gel (42 mL, $0.2 \mathrm{mmol} / \mathrm{mL}$; acetate form) (strong anion exchange, GE Healthcare).

\section{B Lymphoblastoid Cell Culture}

Mono-allelic B lymphoblastoid cells (B-LCL) were purchased from the Fred Hutchinson Cancer Research Center Cell Bank (Seattle, WA). The following cell lines were used: IHW ID IHW01124 HLA-DRB1*01:01; IHW01152 HLA-DRB1*03:01; IHW01111 HLADRB1*04:01; IHW09050 HLA-DRB1*07:01; IHW09036 HLA-DRB1*11:01; and IHW01055*15:01. Cell lines were maintained in RPMI-1640 media (Sigma R0883) 
supplemented with 2 mM L-Glutamine (Sigma G7513), 1 mM sodium pyruvate (Sigma S8636), and 15\% fetal bovine serum (FBS) (Sigma F4135) at $37^{\circ} \mathrm{C}$ in a $5 \% \mathrm{CO}_{2}$ incubator.

\section{B Lymphoblastoid Antigen Pulse}

B-LCLs were cultured in X-Vivo 15 media (Lonza cat\# 04-744Q) supplemented with 10\% human AB serum (HS) (Sigma cat\# H3667) and $5 \mu \mathrm{g} / \mathrm{mL}$ of the human TLR9 ligand CpG ODN 2006 (InvivoGen cat\# tlrl-2006) for $24 \mathrm{~h}$. The following day antigens at the indicated concentrations were added to cells plated to a 6 -well dish at $0.5 \times 10^{\wedge} 6$ cells in $2 \mathrm{~mL}$ of X-Vivo 15 media with $1 \%$ human $\mathrm{Ab}$ serum and $5 \mu \mathrm{g} / \mathrm{mL}$ of CpG ODN 2006. After 24-h incubation, cells were collected for staining with soluble T cell receptors.

Peptides for antigen pulse we generated as a custom reagent by Thermo Fisher Scientific (Waltham, MA). Influenza A H5N1 HA protein (A/Vietnam/1194/2004) was purchased from Sino Biological (cat\# 11062-V08H1).

\section{Primary Dendritic Cell Differentiation and Antigen Pulse}

Two hundred milliliters of whole blood was collected in BD Vacutainer CPT tubes (cat\# 362753) from healthy human donors in the MSD Volunteer Research Donor Program. CD14+ cells were isolated from PBMCs by positive selection using CD14 Microbeads (Miltenyi Biotec cat\# 130-050-201). CD14+ cells were plated to non-adherent 6 well plates at $3 \times$ $10^{\wedge} 6$ cells $/ \mathrm{mL}$ in $3 \mathrm{~mL}$ of in X-Vivo 15 media, $10 \%$ autologous human serum, 1\% PennStrep (Sigma cat\# P4333), IL-4 (1000 IU/mL, R\&D cat\# 204-IL-010/CF), and GM-CSF (1000 IU/mL, R\&D cat\# 7954-GM-050/CF). Cytokines were replenished on days 2 and 4 . Antigens were added to cells on day 4, and DCs were matured with a pulse of LPS ( $1 \mu \mathrm{g} / \mathrm{mL}$, Sigma cat\# L2880). Cells were harvested on day 5 for analysis, and markers for mature DCs were validated by flow cytometry.

\section{Soluble T Cell Receptor Staining and Flow Cytometry Analysis}

Cells were washed with $5 \mathrm{~mL}$ of FACS wash buffer (PBS, $1 \% \mathrm{HS}, 2 \% \mathrm{FBS}$ ) and transferred to a 96-well plate for staining. sTCRs at a final concentration of $500 \mathrm{nM}$ diluted in FACS wash buffer were added to cells for $40 \mathrm{~min}$ at $37^{\circ} \mathrm{C}$ in a final volume of $100 \mu \mathrm{L}$. Following sTCR incubation, cells were washed with $0.5 \mathrm{~mL}$ of PBS once. BioLegend Zombie Aqua cell viability stain (cat\# 423102) diluted 1:500 in PBS was incubated with cells for $10 \mathrm{~min}$ at room temperature. The cell viability stain was not washed off to allow for detection of loss of cell viability during staining. sTCR staining buffer containing mouse anti-His PE secondary antibody (clone J095G46, BioLegend cat\# 362603) at a 1:20 dilution and FcR blocking reagent at a 1:100 dilution (Miltenyi Biotec cat\# 130059-901), and cells were incubated at room temperature for an additional $20 \mathrm{~min}$. Following secondary antibody incubation, cells were washed with $0.5 \mathrm{~mL}$ of FACS wash buffer and cells were resuspended in a final volume of $300 \mu \mathrm{L}$ of FACS wash buffer for analysis. Cells were analyzed immediately using a BD-LSR Fortessa. Staining of $A P C$ Markers. Following $24 \mathrm{~h}$ antigen pulse cell surface marker expression of HLA-DR, CD80, and CD86 was analyzed by flow cytometry. Cells were blocked for $15 \mathrm{~min}$ at $4{ }^{\circ} \mathrm{C}$ in FACS wash buffer containing a 1:100 dilution of FcR blocking reagent. Mouse anti-human CD86 (BV421) (clone 2331, BD Biosciences cat\# 562433) at a 1:100 dilution, mouse antihuman CD80 (PE) (clone L307.4, BD Biosciences cat\# 557227) at a 1:20 dilution, and mouse anti-human HLA-DR (APC-H7) (clone G46-6, BD Biosciences cat\# 561358) at a 1:100 dilution were added to cells in a final volume of $100 \mu \mathrm{L}$ for $20 \mathrm{~min}$ at $4^{\circ} \mathrm{C}$ in FACS wash buffer with FcR blocking reagent. As a control, cells were incubated with isotype control Abs at the same dilution: BV421 mouse IgG1, k (clone X40, BD Biosciences cat\# 562438), PE mouse IgG1, k (clone MOPC-21, BD Biosciences cat\# 555749), and APC-H7 Mouse IgG2a, k (clone G155-187, BD Biosciences cat\# 560897). Following staining, cells were washed with FACS wash buffer and resuspended $300 \mu \mathrm{L}$ of FACS wash buffer containing 7AAD viability dye at 1:100 dilution for analysis (eBioscience cat\# 00-6993). Cells were not fixed and analyzed immediately using a BD-LSR Fortessa.

Data was analyzed using FlowJo (version 10). Cells were gated according to their forward and side scatter properties and single cells were selected by comparison of FSC-A to FSC-H. Dead cells were excluded from analysis. A representative gating strategy can be found in S2 Fig.

\section{Chloroquine Inhibition}

Chloroquine (Sigma cat\# C6628) at $80 \mu \mathrm{M}$ was added to cells $1 \mathrm{~h}$ prior to addition of antigens. Cells were cultured in the presence of chloroquine and antigens for $24 \mathrm{~h}$ before sTCR binding analysis. As the effects of chloroquine are quickly reversible, chloroquine at $20 \mu \mathrm{M}$ was added to the FACS wash buffer in treated cells.

\section{MAPPs Assay}

A total of $1 \times 10^{\wedge} 7$ cells per sample were submitted to Caprion Biosciences Inc. (Montreal, Quebec, CA) for MAPPs analysis. MHC class II immunoprecipitation and LS/MS-MS analysis were performed by Caprion Biosciences Inc. according to the method previously published by Mommen et al. (9). Primary DCs and B-LCLs were pulsed with Omomyc $(10 \mu \mathrm{g} / \mathrm{mL}, 0.5 \mu \mathrm{M})$ under conditions for antigen pulse described above. Mock (vehicle control) cells were included as a control.

\section{In Silico Predictions}

The 90AA long Omomyc sequence was divided into 9 peptides of 25AA in length with 10AA overlap. MHC Class II binding affinity of these peptides to HLA-DRB1*11:01 or HLA-DRB1*15:01 was analyzed using the following algorithms: IEDBcons (20), Epimatrix (Epivax) (21), NN_Align (22), or NetMHC3.2 (23). The number of unique core binding epitopes within the top $5 \%$ binding rank for Epimatrix and top $10 \%$ binding rank for IEDBcons, NN_Align, and NetMHC3.2 was considered as HLA-DRB1 binders. 


\section{Data Presentation and Statistical Analysis}

Graphpad prism (version 8) was used for analysis. In Fig. 2, data is expressed as the fold MFI of sTCR fluorescence in peptide-treated cells of mock-treated cells: $\mathrm{MFI}_{(\text {reference }}$ epitope) / median $\mathrm{MFI}_{(\text {mock })}$. One-way ANOVA with multiple comparisons was used to determine significance, $p>0.05$ not significant (ns). In Figs. 3 and $4 \mathrm{~b}$, data is expressed as the percent sTCR florescence signal (MFI) relative to cells pulsed with reference epitope: $\mathrm{MFI}_{\text {(test compound }+ \text { reference epitope) }} /$ median $\mathrm{MFI}_{\text {(reference epitope alone) }}$. In Fig. 5, data is expressed as the percent sTCR positive relative to cells pulsed with reference epitope: \%sTCR+ ${ }_{(\text {test }}$ compound + reference epitope) $/$ median $\% s T C R+{ }_{(\text {reference epitope alone). }}$ One-way ANOVA with multiple comparisons was used to compare reference peptide to test compound + reference peptide $p>0.05$ not significant $(n s)$. Bars represent median and IQR of triplicate samples.

\section{RESULTS}

\section{A Novel In Vitro MHC Class II Presentation Assay Employing Soluble T Cell Receptors}

We describe a novel cell-based assay for MHC class II epitope processing and presentation that employs fluorescently labeled sTCRs for detection of pMHC. Antigenspecific sTCRs have been developed as novel therapeutics for immune modulation and targeted drug delivery $(18,19$, 24). We employed the method established by Walseng et al. for the generation of soluble $\mathrm{T}$ cell receptors in mammalian cells (17). For expression, a vector containing TCR alpha and beta chains with the transmembrane and cytoplasmic domain removed was transfected into mammalian cells (17) (Fig. 1a). Production in mammalian cells offers benefits over bacteria in allowing for post translational modifications. A picornavirus ribosomal skipping sequence $2 \mathrm{~A}$ was included between TCR alpha and beta chains allowing for equimolar production of both chains and higher efficiency of pairing (25-27).

Two soluble TCRs were generated. Sequences of the variable and constant domain were utilized from the F24 TCR binding the HIV-1 capsid epitope Gag293 (p24) in the context of HLA-DRB1*01:01, HLA-DRB1*11:01, and HLADRB1*15:01 (28). A second TCR (F11) recognizing the influenza hemagglutinin epitope HA306 (HA) in the context of HLA-DRB1*01:01 was also expressed (29). Expression of TCR construct in mammalian cell lines produced a secreted protein dimer that separated under reducing conditions (S1 Fig). Peptides of the HIV-capsid epitope Gag293 (p24) and influenza hemagglutinin epitope HA306 (HA) will serve as reference epitopes since they can be detected by the F24 and F11 sTCRs respectively when presented by MHC-II.

To assess if any peptide or protein contains epitopes, defined as sequences presented on a specific HLA-DR allele, B lymphoblastoid cell lines (B-LCL) mono-allelic for the HLA-DRB1 locus were pulsed with the peptide or protein of interest (referred as test-peptide and test-compound respectively) and a reference epitope (Fig. 1b). A labeled sTCR specific to the $\mathrm{e}_{\mathrm{r}} \mathrm{MHC}$ was used to quantitate reference epitope presentation by flow cytometry. Since epitopes restricted by the same $\mathrm{MHC}$ molecule compete for binding (16), efficient presentation of the test-peptide will reduce the level of $\mathrm{e}_{\mathrm{r}} \mathrm{MHC}$ detected by the labeled sTCR (Fig. 1b). Comparing the signal obtained when cells are pulsed with a reference epitope alone versus cells pulsed with a reference epitope and a test-peptide will indicate if the test-peptide contains epitopes presented on the HLA-DR allele (Fig. 1b). Decreased sTCR binding caused by competition will be quantified as the percent reduction in fluorescent signal: (reference epitope + test compound) / (median of reference epitope alone). The main advantage of this competitionbased approach is that only a single sTCR needs to be generated for a given HLA-reference peptide complex. Using this strategy, any protein can be analyzed to determine if it contains epitopes presented on a HLA allele. Furthermore, the use of mono-allelic antigen presenting cell lines avoids additional steps required for allelic deconvolution.

\section{sTCRs Specifically Bind the Reference Epitope in Context of Its Cognate MHC Class II}

To test if the sTCRs can bind the $\mathrm{e}_{\mathrm{r}} \mathrm{MHC}$ complex, BLCLs were incubated with increasing concentrations of influenza HA reference epitope or HIV p24 reference epitope for $24 \mathrm{~h}$. Fluorescently labeled sTCRs were employed to assess epitope presentation. Relative to mock (vehicle control), a dose-dependent increase in bound F24 p24 sTCR was observed in HLA-DRB1*11:01 and *15:01 when pulsed with the p24 epitope (Fig. 2a). Cross-recognition of the p24 epitope by F24 TCR in the context of multiple HLA-DR alleles has been previously demonstrated (28). Similarly, the F11 HA sTCR bound HLA-DRB1*01:01 and *04:01 cells when pulsed with the HA epitope (Fig. 2b). Even though F11 is reported to bind the HA epitope in the context of HLADRB1*01:01 only (29), F11 cross-recognized the HA epitope when presented on HLA-DRB*04:01 (Fig. 2a). Similar to cross-recognition of the F24 sTCR in the context of multiple HLA types, cross-recognition of HA epitope in the context of HLA-DRB1*01:01 and *04:01 by the HA1.7 TCR has also been observed (30). It is therefore not surprising that the HAspecific F11 sTCR was able to recognize the HA epitope in the context of both HLA-DRBB*01:01 and *04:01 in our assay. Because mono-allelic cell lines are used, crossrecognition will not confound the output of this assay. Furthermore, the F11 sTCR was also able to detect the HA epitope presentation on HLA-DRB1*01:01 expressing primary DCs (S3A Fig).

To investigate the specificity of sTCR/ $\mathrm{e}_{\mathrm{r}} \mathrm{MHC}$ binding, BLCL were pulsed with either the p24 epitope or the HA epitope and stained with sTCRs (Fig. 2c and d). F24 sTCR stained HLA-DRB1*11:01 and HLA-DRB1*15:01 when pulsed with the p24 epitope only (Fig. 2c). F11 sTCR stained HLA-DRB1*01:01 and *04:01 expressing cells when pulsed with the HA epitope only (Fig. 2d). A low signal was detected when F24 sTCR was used to stain HA-epitope pulsed HLADRB1*15:01 expressing cells. However, the F24 was significantly better at staining p24-epitope HLA-DRB1*15:01 expressing cells (Fig. 2c). Both the p24 and the HA epitopes are promiscuous epitopes, able to bind multiple HLA-DR1 alleles, and therefore can be presented on all HLA types tested $(28,30)$.

The concentration of peptide input required for significant sTCR binding varied between cell lines, ranging from a 
a

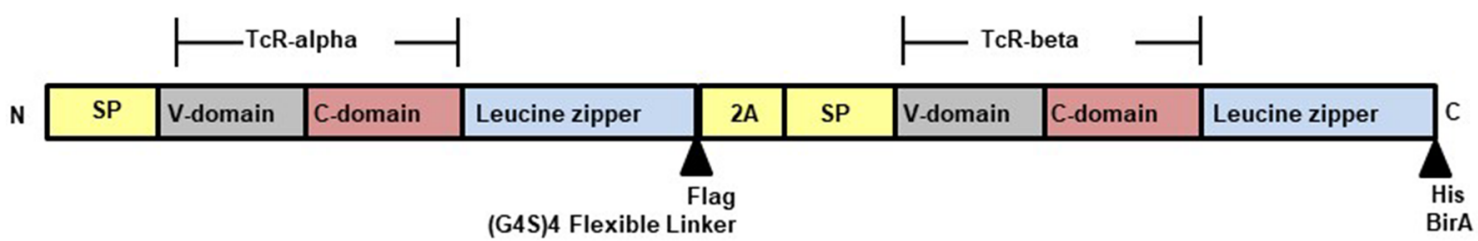

b
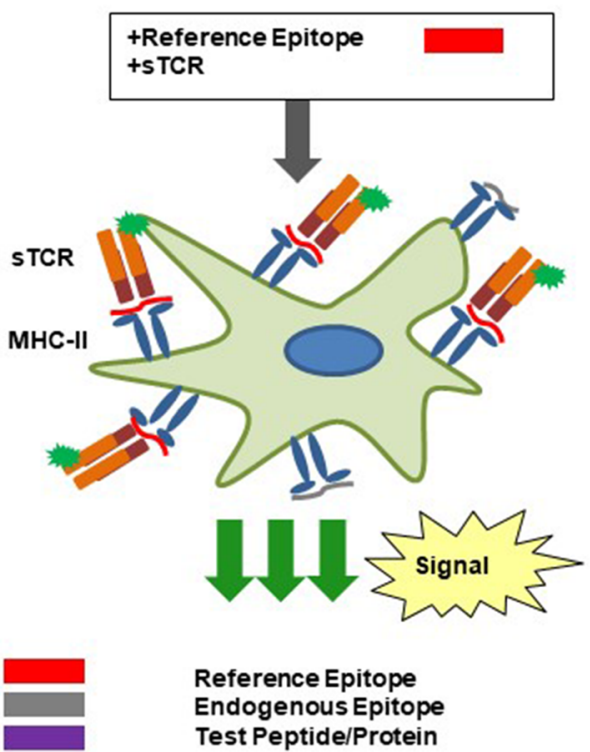

Reference Epitope Endogenous Epitope Test Peptide/Protein

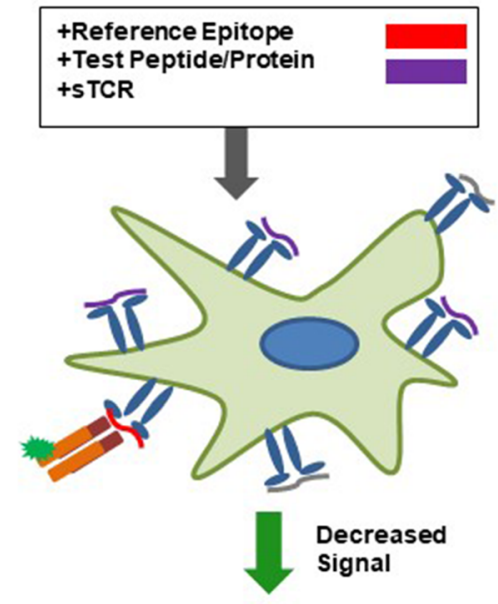
ptide/Protein

Fig. 1. Soluble $T$ cell receptor construct and assay design. a Construct design of truncated soluble TCRs: a single ORF encoding TCR $\alpha$ and TCR $\beta$ variable and constant domains and leucine zipper followed by a flexible (G4S)4 linker for interchain stability, native signal peptide (SP) was used. Picornavirus ribosomal skipping 2A sequence allows for equimolar chain production (2A). b Assay design: cell-based assay to assess antigen presentation. Left panel: cells are pulsed with reference epitope only. Right panel: cells are pulsed with reference epitope and a test peptide/protein. Efficient test compound presentation is quantified by decreased sTCR binding signal to the reference antigen

minimum detection limit of $1 \mu \mathrm{M}$ for $\mathrm{p} 24$ in the HLA-DRB1*11:01 line to $400 \mu \mathrm{M}$ HA in the HLA-DRB1*01:01 line (Fig. 2a and b). F24 TCR binds with the highest affinity to HLA-DRB1*11:01 expressing cells, followed by HLA-DRB1*15:01 and HLADRB1*01:01 expressing cells (28). Our analysis supports these findings, with a median 21 -fold increase in binding relative to mock in HLA-DRB1*11:01 expressing cells when pulsed with $100 \mu \mathrm{M}$ of the p24 epitope, versus a 2.5 -fold increase in binding relative to mock observed when HLA-DRB1*15:01 expressing cells were pulsed with the same amount of the p24 epitope (Fig. 2a). Furthermore, HLA-DR expression levels were consistent between the different cell lines and were not affected when these cells were pulsed with the different epitopes (S4 Fig). As TCR/ MHC and peptide affinity differs between HLA types, assay conditions such as concentration of the reference epitope and the sTCR will need to be optimized for each HLA allele and sTCR.

\section{Competition-Mediated Detection of Test Epitope Presentation on MHC-II}

To investigate the assay's ability to detect MHC class II presentation of a test antigen by competing out the binding of the reference epitope, APCs were pulsed with the reference epitope alone or in the presence of an increasing concentration of test compound. APCs were subsequently stained with sTCR specific for the $\mathrm{e}_{\mathrm{r}} \mathrm{MHC}$ (Fig. 3a). F24 was used to stain B-LCLs pulsed with the p24 epitope as reference epitope alone or with an increasing concentration of the HA epitope as test peptide (Fig. 3a). Likewise, F11 was used to stain B-LCLs and primary human DCs pulsed with the HA epitope as a reference epitope alone or with an increasing concentration of p24 epitope as test peptide (Fig. 3b and S3B Fig). A significant dose-dependent reduction in reference epitope detection was observed indicating successful presentation of the test peptide (Fig. 3a and b). This is expected since both epitopes (p24 and HA) employed in this experiment are able to bind multiple HLA-DR alleles $(28,30)$.

We next investigated if this assay could detect epitopes in a context of a full protein antigen. Omomyc a 90AA dimer (31) or influenza H5N1 HA a 522AA trimer (32) was used as test compounds in an experiment employing the p24 epitope as a reference epitope and the F24 sTCR (Fig. 3c and d). Both Omomyc and H5N1 HA significantly reduced sTCR detection of the p24 reference antigen (Fig. 3c and d). This indicates that both proteins contain epitopes able to bind the specific HLA-DR alleles expressed by BLC-Ls, and these cell lines are capable of processing and presenting epitopes in the context of full proteins and not just peptides. 
a

9036 HLA-DRB1 11:01

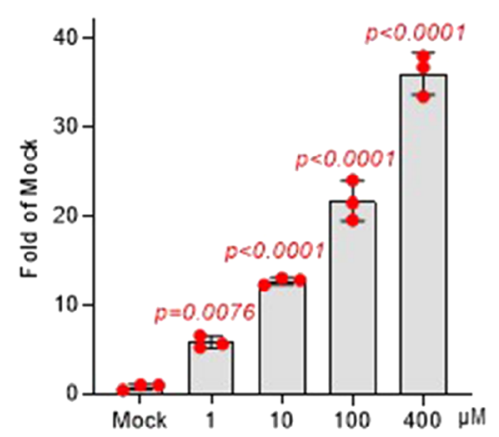

c

1111 HLA-DRB1 04:01

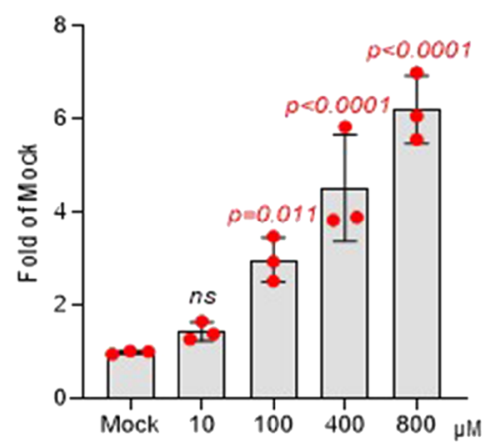

1055 HLA-DRB1 15:01

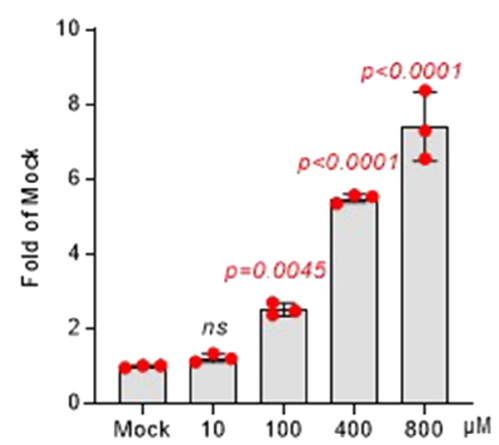

1124 HLA-DRB1 01:01

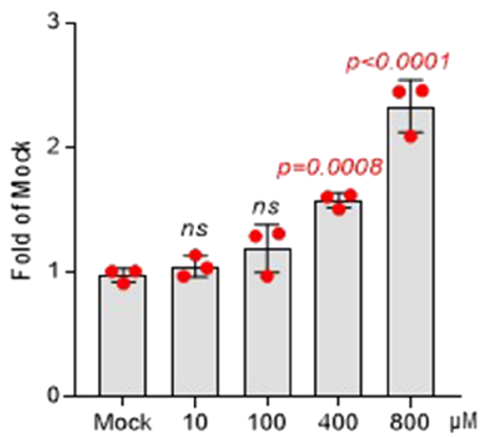

b
1055 HLA-DRB1 15:01

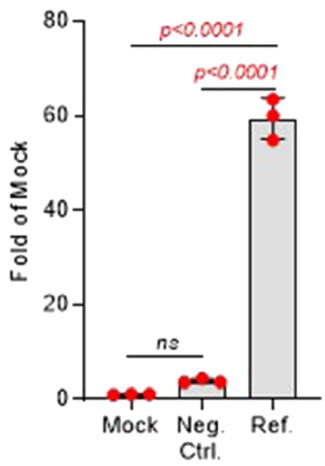

d

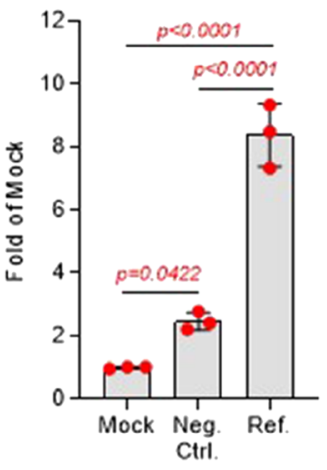

124 HLA-DRB1 01:01

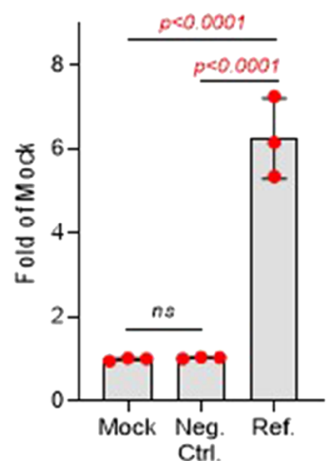

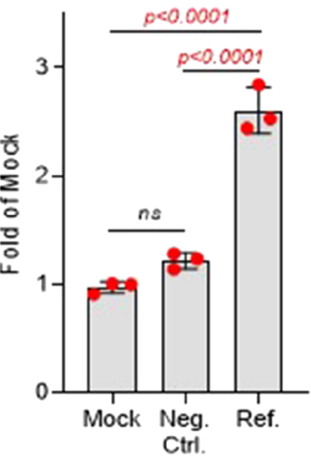

Fig. 2. sTCRs specifically bind the reference peptide and MHC class II complex. a F24 staining of 9036 HLA-DRB1*11:01 and 1055 *15:01 cells pulsed with an increasing amount of p24 epitope ( $X$-axis). b F11 staining of 1111 HLA-DRB1*04:01 and 1124 *01:01 cells pulsed with an increasing amount of the HA epitope ( $X$-axis). c F24 staining of 9036 HLA-DRB1*11:01 and 1055*15:01 cells pulsed with 1200 $\mu$ M of the reference epitope (p24) or a negative control (HA). d F11 staining of 1111 HLA-DRB1*04:01 and 1124 *01:01 cells pulsed with 1200 $\mu$ M of the reference epitope (HA) or a negative control (p24). The data on Y-axis is expressed as the fold MFI of sTCR fluorescence in peptide-treated

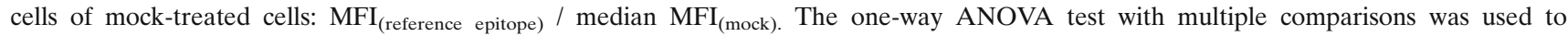
determine significance, $p>0.05$ not significant $(n s)$

Epitope Mapping Using Overlapping Peptides and Correlation with In Silico Predictions and MAPPs Assay Outputs

Following the observation that the Omomyc protein antigen contains HLA-DR binding epitopes (Fig. 3b), a strategy of overlapping peptides was devised to map these epitopes. Nine peptides of 25AA in length with 10AA overlap were generated to cover the length of the Omomyc protein (Fig. 4a). B-LCLs were pulsed with p24 reference epitope alone or in combination with each of the 9 overlapping Omomyc peptides and stained with the F24 sTCR (Fig. 4b). Reduced sTCR/ $/ \mathrm{e}_{\mathrm{r}} \mathrm{MHC}$ binding was observed in the presence of peptides spanning AA 27-70 in HLA-DRB1*11:01 expressing cells, and AA 36-61 in HLADRB1*15:01 expressing cells indicating the presence of MHC class II binding epitopes (Fig. 4b).

To compare the output of this assay with in silico epitope prediction tools, we analyzed the Omomyc sequence for peptide/MHC class II binding using four MHC-II binding prediction algorithms: IEDBcons (33), Epimatrix (21), NN_Align (22), and NetMHC3.2 (23). The number of unique core binding epitopes was quantified using the best practices for each algorithm and indicated as "hits" which ranged from 0 to 4 epitopes (Fig. 4c). When comparing outputs, consensus between all 4 prediction algorithms was observed only for peptides detected by the in vitro sTCR assay (Fig. 4c). In silico tools predicted the presence of additional epitopes not presented in the in vitro sTCR assay, this could be due to overprediction associated with in silico immunogenicity tools as reported before (34). Next, we compared epitope presentation delineated by the sTCR-based assay versus the standard MAPPs in vitro APC assay. To perform this analysis, four B-LCL lines expressing distinct HLA-DRB1 alleles (HLA-DRB1*01:01; *03:01, *07:01, or *15:01) were pulsed with full length Omomyc for $24 \mathrm{~h}$ (Fig. 4d). Peptide sequences bound to MHC were eluted and sequences were determined by mass spectrometry. MHC class II bound sequences determined by MAPPs were compared with Omomyc peptides detected to bind in the sTCR assay (Fig. 4b). Epitopes were identified by MAPPs spanning AA position 29-77. This region overlapped with peptides detected by the sTCR assay which covered AA position 27-70 for HLA-DRB1*11:01 and AA 36-61 for HLA-DRB1*15:01 (Fig. 4d). Although HLA types varied between assays, the detected core binding epitopes were consistent. These core epitopes located at AA 
a

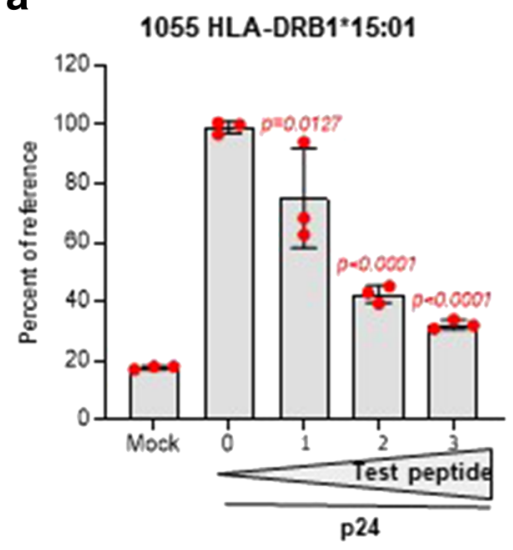

b

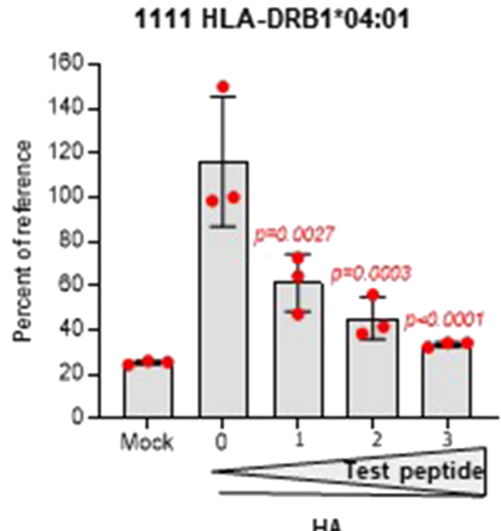

9036 HLA-DRB1*11:01

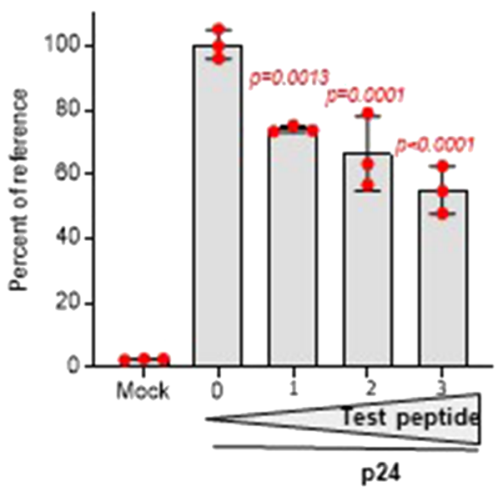

1124 HLA-DRB1*01:01

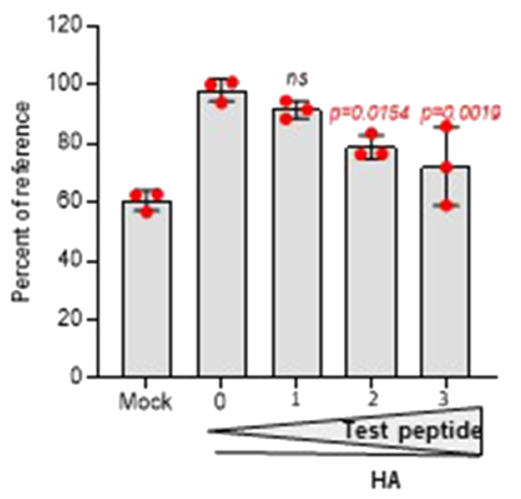

C
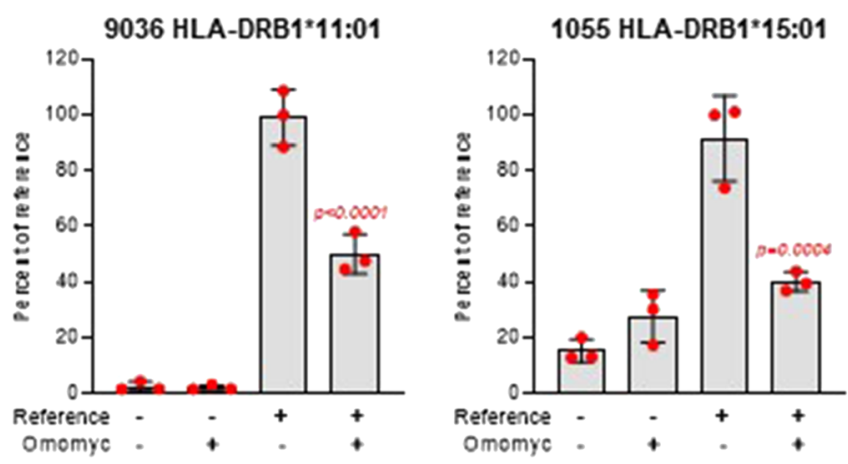

d

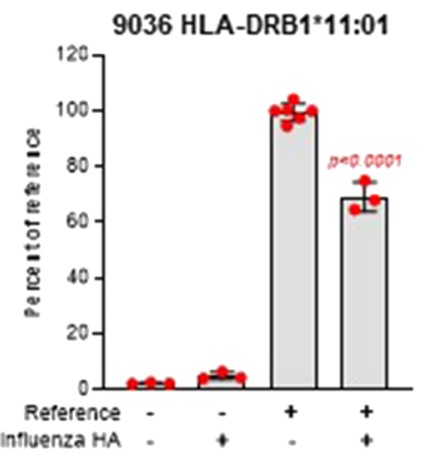

Fig. 3. Competition-mediated detection of test epitope presentation on MHC-II. a F24 staining of 1055 HLA-DRB1*15:01 and 9036*11:01 pulsed with p24 (reference epitope) alone or with an increasing concentration of HA test antigen (test peptide). b F11 staining of 1111 HLA-DRB1*04:01 and 1124*01:01 cells pulsed with HA (reference epitope) alone or with an increasing concentration p24 test antigen (test peptide) (c, d). F24 staining of 9036 HLA-DRB1*11:01 and 1055*15:01 cells was pulsed with p24 alone or in the presence of Omomyc (c) or influenza A H5N1 HA protein (d). The data on $Y$-axis is expressed as the percent sTCR florescence signal (MFI) relative to cells pulsed with reference epitope: MFI $_{\text {(test compound }}$ + reference epitope) / median $\mathrm{MFI}_{\text {(reference epitope alone). The one-way ANOVA with multiple comparisons was }}$ used to determine significance, $p>0.05$ not significant ( $n s)$. For statistical comparisons in $\mathbf{a}$ and $\mathbf{b}$, samples treated with test peptide were compared to no test epitope; for $\mathbf{c}$ and $\mathbf{d}$, samples treated with reference peptide + protein were compared to the reference epitope alone

positions 43, 46, and 53 represent promiscuous epitopes, binding with high affinity to all included HLA types (Fig. 4b and d). MAPPs detected an epitope spanning AA position 70-77 in HLA-DRB1*03:01; *07:01; and *15:01 cells (Fig. 4d). A non-significant trend of decreased $e_{\mathrm{r}} \mathrm{MHC} / \mathrm{sTCR}$ binding was observed when HLA-DRB1*15:01 expressing cells were pulsed with peptides spanning AA region 63-90 (Fig. 4b). Finally, we compared MAPPs detected epitopes between B-LCLs and primary DCs when pulsed with the full Omomyc protein. Both B-LCLs and primary DCs presented 
a

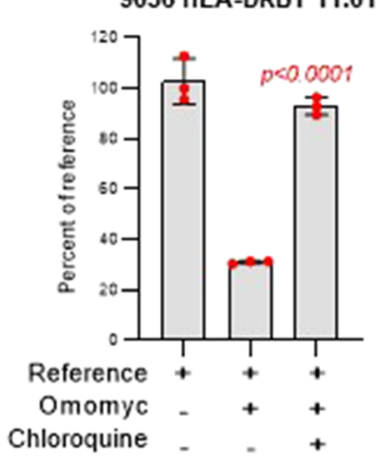

1055 HLA-DRB1“15:01

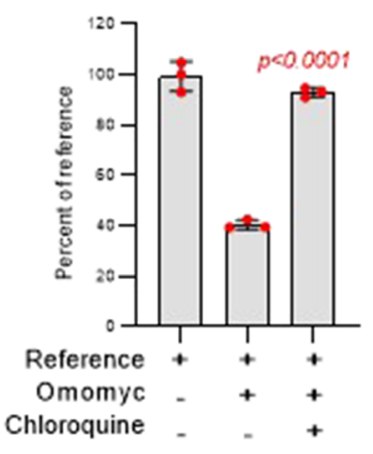

b
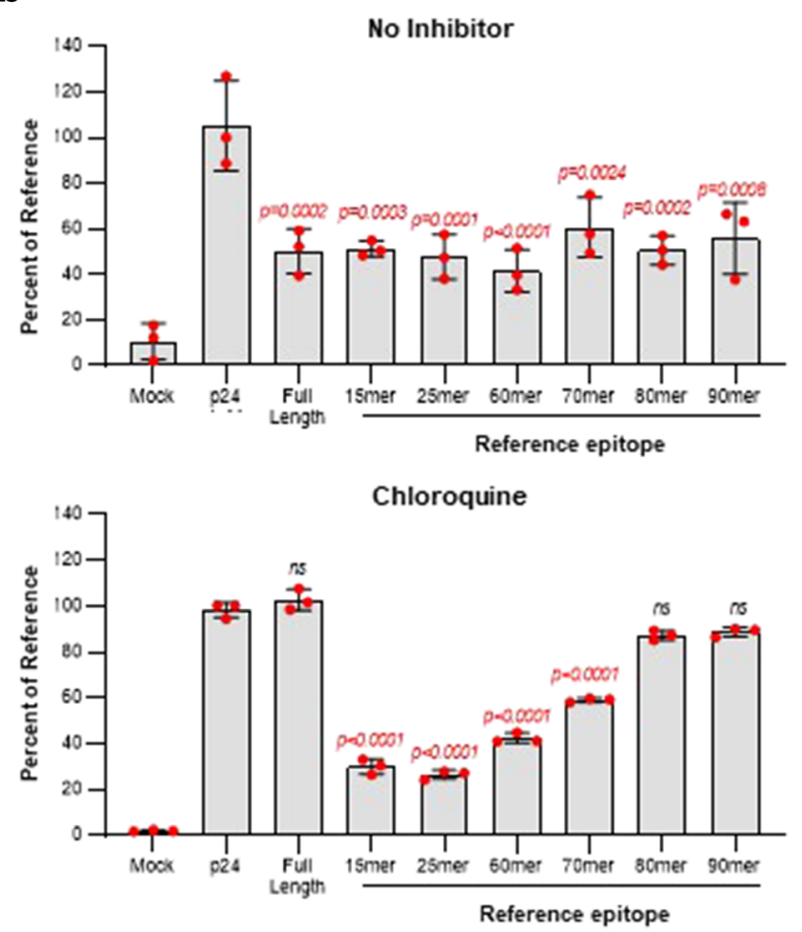

Fig. 5. Minimum peptide length required for endosomal processing. a F24 binding to p24/MHC-II complex in the presence full length Omomyc in untreated B-LCLs or B-LCLs treated with chloroquine. b F24 binding to p24/MHC-II complex in B-LCLs pulsed with Omomyc peptides of increasing lengths in the presence or absence of chloroquine. The data on $Y$-axis is expressed as the percent sTCR florescence signal (MFI) relative to cells pulsed with reference epitope: $\mathrm{MFI}_{\text {(test compound + reference epitope) } / \text { median } \mathrm{MFI}_{\text {(reference }}}$ epitope alone). The one-way ANOVA with multiple comparisons was used to determine significance, $p>0.05$ not significant $(n s)$

Chloroquine had no impact on the ability of Omomyc peptides to compete with the reference epitope when the competing peptide length was shorter than 70AA (Fig. 5b). For peptide length ranging from 80 to $90 \mathrm{AA}$, chloroquine was able to inhibit the ability of the encoded epitopes to compete the reference epitope (Fig. 5b compare top and bottom panel). In the absence of chloroquine, 80-90mer Omomyc peptides reduced sTCR binding to p24/HLA-DR1 complex by $50-60 \%$ (Fig. $5 \mathrm{~b}$ top panel), where in the presence of chloroquine, no significant reduction of sTCR binding to p24/ HLA-DR1 was observed.

\section{DISCUSSION}

Here we present a proof of concept for a novel in vitro assay capable of assessing MHC-II antigen processing and presentation and promises an accurate, fast, and cost-effective turnaround. This assay employs fluorescently labeled soluble TCRs recognizing MHC class II/epitope complex as a novel approach for assessing antigen presentation. In addition, epitope competition for MHC binding minimizes the number reagents required for this assay, as only a single sTCR and reference epitope are needed for each MHC supertype. We propose to employ APCs mono-allelic for the MHC locus to eliminate the ambiguity that arises from the co-expression of multiple MHC alleles; however, this assay was also able to assess antigen presentation in multi-allelic human primary DCs (S3A Fig).

An integral component of this assay is the concept of epitope competition for MHC antigen presentation (16). Peptide sequences as small as $15 \mathrm{AA}$ in length and full proteins up to 522AA were able to compete with the reference antigen for MHC class II presentation (Fig. 3a-c). The concept of competition was described by Adorini et al., who detected decreased antigen-specific $\mathrm{T}$ cell activation when hen egg-white lysozyme-transfected APCs were cultured with exogenous peptides (16). Sensitivity of sTCR in detecting reference antigen was highly dependent on the affinity of the TCR/eMHC complex. The detection limit for the high affinity F24 TCR was $1 \mu \mathrm{M}$ of p24 when HLADRB1*11:01 expressing cells were used; on the other hand, F11 sTCR required $400 \mu \mathrm{M}$ of HA peptide in HLADRB1*01:01 expressing cells. Low sensitivity can be easily overcome by selecting sTCR with a higher affinity for eMHC complex or multimerizing the sTCR to increase the avidity of eMHC/sTCR interaction $(37,38)$. Alternatively, high affinity TCR mimic antibodies or TCR engagement reporter cells can be used (39-43). The competition-based assay described here enabled the detection of competing epitopes, despite differences of sTCR sensitivity to the reference antigen. These analyses were able to consistently detect competition by high affinity antigens and epitopes. Furthermore, since the strength of MHC peptide binding affinity was correlated with immunogenicity $(44,45)$, the ability to detect high affinity epitopes is integral in identifying immunogenicity risks.

When comparing epitope presentation on B-LCLs versus primary DCs, we observed high alignment of presented epitopes between the sTCR assay and the standard MAPPs assay (Figs. 4b and S3). The application of B-LCLs as APCs capable of inducing antigen-specific $\mathrm{CD} 4+\mathrm{T}$ cell activation has been widely established (46-50). While B-LCLs are utilized as the model APC in our study, the assay described here can be easily adapted to other APCs. Macrophage and DC cell lines as well as primary human APCs could be easily employed as epitope competition and detection by STCR binding are not B-LCL dependent.

Similar binding specificities enabled clustering of HLADR alleles into eight super types (44). In this study, we employed four cell lines that represent four HLA II super types: HLA-DRB1*01:01, HLA-DRB1*04:01, HLADRB1*11:01, and HLA-DRB1*15:01. To achieve broad coverage, inclusion of additional HLA-DR super types as well as HLA-DQ and HLA-DP alleles will be required. The 
assay described here is not allele nor class specific, and the binding profile of any HLA can be determined as long as a sTCR specific for a reference epitope can be generated.

The B-LCLs employed here were capable of presenting epitopes from full-length proteins (Fig. 3b). Antigen processing by B-LCLs was inhibited by chloroquine, an inhibitor of endosomal proteases used as a correlate for endosomal processing (Fig. 5) (36). To map the specific position of epitopes within a protein sequence, a strategy of overlapping peptides is required. The sTCR assay enabled an overlapping peptide strategy spanning the entire Omomyc length to map the position of epitopes within the Omomyc protein (Fig. 4a and b). The sTCR assay detected epitopes which overlapped with those detected standard MAPPs assay (Fig. 4d). Furthermore, the sTCR assay was able to identify overprediction implied by in silico tools (Fig. 4c). MAPPs detected presentation of Omomyc AA 61-77 in 1055 HLADRB1*15:01 which was not detected by the sTCR assay. This discrepancy could be attributed to weaker affinity to the sTCR/peptide complex to p24/HLA-DRB1*15:01 leading to decreased sensitivity of detection. Methods for optimizing sTCR/reference peptide affinity could also further increase accuracy.

A caveat with employing peptides in cell-based antigen presentation assays is highlighted by Sorde et. al, where a MAPPs assay identified an epitope when presented in the context of a peptide but not the full parent protein (35). This observation implies that unlike full length proteins, endosomal processing is not required for peptide encoded epitopes. To overcome this limitation, we identified the minimal peptide size requiring endosomal processing prior to epitope binding in the B-LCLs. Chloroquine decreased the ability of full length Omomyc to compete with the reference epitope (Fig. 5). Chloroquine treatment increased the presentation of the reference p24 epitopes which is a 15mer peptide (S6 Fig). When cells were pulsed with peptides of increasing length, pretreatment of cells with chloroquine affected the presentation of epitope encoded in peptide of 80-90AA in length (Fig. 5b). This indicates that at least for the B-LCLs used in this study, peptides with a minimum of $80 \mathrm{AA}$ in length should be employed to ensure endosomal processing.

\section{CONCLUSIONS}

This work describes soluble $\mathrm{T}$ cell receptors that can recognize an epitope/MHC class II complex in the context of four major MHC classes: HLA-DRB1*01:01, *04:01, *11:01, and *15:01. In summary, we describe a novel in vitro assay for analysis of antigen processing and presentation in the context of a specific MHC class II molecule. The described assay has the potential for detecting antigen presentation by multiple APC types within the context of both MHC classes I and II.

\section{ACKNOWLEDGMENTS}

We thank Shiying Chen and Claudio Mapelli for providing the Omomyc compound, Robert Xiao and his team at GenScript Biotech for generating soluble TCRs, and Mark Watson and his team at Caprion for performing MAPPs analysis.

\section{AUTHOR CONTRIBUTIONS}

CT and JP performed the experiments. CT, VJ, and JM contributed to experimental design, data discussion, and manuscript preparation. CT and JM wrote the paper. JM oversaw the entire project. All authors read and approved the final manuscript.

\section{FUNDING}

The authors thank the Merck Research Laboratories Postdoctoral Research Fellows Program for funding and supporting this work.

\section{SUPPLEMENTARY INFORMATION}

The online version contains supplementary material available at https://doi.org/10.1208/s12248-020-00553-x.

\section{REFERENCES}

1. McHeyzer-Williams LJ, McHeyzer-Williams MG. Antigenspecific memory B cell development. Annu Rev Immunol. 2005;23:487-513.

2. Roche PA, Furuta K. The ins and outs of MHC class IImediated antigen processing and presentation. Nat Rev Immunol. 2015;15(4):203-16.

3. Huppa JB, Davis MM. T-cell-antigen recognition and the immunological synapse. Nat Rev Immunol. 2003;3:973-83.

4. Kurosaki T, Kometani K, Ise W. Memory B cells. Nat Rev Immunol. 2015;15:149-59.

5. Jawa V, Cousens LP, Awwad M, Wakshull E, Kropshofer H, De Groot AS. T-cell dependent immunogenicity of protein therapeutics: preclinical assessment and mitigation. Clin Immunol. 2013;149:534-55.

6. De Groot AS, Scott DW. Immunogenicity of protein therapeutics. Trends Immunol. 2007;28(11):482-90.

7. Moser JM, Sassano ER, Leistritz DC, Eatrides JM, Phogat S, Koff $\mathrm{W}$, et al. Optimization of a dendritic cell-based assay for the in vitro priming of naïve human $\mathrm{CD} 4+\mathrm{T}$ cells. J Immunol Methods. 2010;353:8-19.

8. Schlienger K, Craighead N, Lee KP, Levine BL, June $\mathrm{CH}$. Efficient priming of protein antigen-specific human CD41 $\mathrm{T}$ cells by monocyte-derived dendritic cells. Blood. 2000;96(10):3490-8.

9. Mommen GPM, Marino F, Meiring HD, Poelen MCM, van Gaans-van den Brink JAM, Mohammed S, et al. Sampling from the proteome to the human leukocyte antigen-DR (HLA-DR) ligandome proceeds via high specificity. Mol Cell Proteomics. 2016;15(4):1412-23.

10. Abelin JG, Keskin DB, Sarkizova S, Hartigan CR, Zhang W, Sidney J, et al. Mass spectrometry profiling of HLA-associated peptidomes in mono-allelic cells enables more accurate epitope prediction. Immunity. 2017;46(2):315-26.

11. Sekiguchi N, Kubo C, Takahashi A, Muraoka K, Takeiri A, Ito $\mathrm{S}$, et al. MHC-associated peptide proteomics enabling highly sensitive detection of immunogenic sequences for the development of therapeutic antibodies with low immunogenicity. MABS. 2018;10:1168-81.

12. Nielsen M, Lund O, Buus S, Lundegaard C. MHC class II epitope predictive algorithms. Immunology. 2010;130:319-28.

13. Chen B, Khodadoust MS, Olsson N, Wagar LE, Fast E, Liu CL, et al. Predicting HLA class II antigen presentation through integrated deep learning. Nat Biotechnol. 2019;37:1332-43.

14. Schneidman-Duhovny D, Khuri N, Dong GQ, Winter MB, Shifrut E, Friedman N, et al. Predicting CD4 T-cell epitopes 
based on antigen cleavage, MHCII presentation, and TCR recognition. PLoS One. 2018;13:1-22.

15. Barra C, Alvarez B, Paul S, Sette A, Peters B, Andreatta M, et al. Footprints of antigen processing boost MHC class II natural ligand predictions. Genome Med. 2018;10:1-15.

16. Adorini L, Moreno J, Momburg F, Hammerling GJ, Guery JC, Valli A, et al. Exogenous peptides compete for the presentation of endogenous antigens to major histocompatibility complex class II-restricted T cells. J Exp Med. 1991;174(4):945-8.

17. Walseng E, Walchli S, Fallang LE, Yang W, Vefferstad A, Areffard A, et al. Soluble T-cell receptors produced in human cells for targeted delivery. PLoS One. 2015;10(4):e0119559.

18. Card KF, Price-Schiavi SA, Liu B, Thomson E, Nieves E, Belmont $\mathrm{H}$, et al. A soluble single-chain T-cell receptor IL-2 fusion protein retains MHC-restricted peptide specificity and IL-2 bioactivity. Cancer Immunol Immunother. 2004;53:345-57.

19. Molloy PE, Sewell AK, Jakobsen BK. Soluble T cell receptors: novel immunotherapies. Curr Opin Pharmacol. 2005;5:438-43.

20. Garfall AL, Maus MV, Hwang WT, Lacey SF, Mahnke YD, Melenhorst JJ, et al. Chimeric antigen receptor T cells against CD19 for multiple myeloma. N Engl J Med. 2015;373:1040-7.

21. De Groot AS, Martin W. Reducing risk, improving outcomes: bioengineering less immunogenic protein therapeutics. Clin Immunol. 2009;131:189-201.

22. Nielsen M, Lund O. NN-align. An artificial neural networkbased alignment algorithm for MHC class II peptide binding prediction. BMC Bioinf. 2009;10:296-306.

23. Jensen KK, Andreatta M, Marcatili P, Buus S, Greenbaum JA, Yan Z, et al. Improved methods for predicting peptide binding affinity to MHC class II molecules. Immunology. 2018;154(3):394-406.

24. Oates J, Hassan NJ, Jakobsen BK. ImmTACs for targeted cancer therapy: why, what, how, and which. Mol Immunol. 2015;67:67-74.

25. Walchli S, Loset GA, Kumari S, Johansen JN, Yang W, Sandlie I, et al. A practical approach to T-cell receptor cloning and expression. PLoS One. 2011;6(11):e27930.

26. Szymczak AL, Workman CJ, Wang Y, Vignali KM, Dilioglou S, Vanin EF, et al. Correction of multi-gene deficiency in vivo using a single 'self-cleaving' $2 \mathrm{~A}$ peptide-based retroviral vector. Nat Biotechnol. 2004;22(5):589-94.

27. de Felipe P, Luke GA, Hughes LE, Gani D, Halpin C, Ryan MD. E unum pluribus: multiple proteins from a self-processing polyprotein. TRENDS Biotehnol. 2006;24:68-75.

28. Galperin M, Farenc C, Mukhopadhyay M, Jayasinghe D, Decroos A, Benati D, et al. CD4+ T cell-mediated HLA class II cross-restriction in HIV controllers.pdf. Sci Immunol. 2018:112.

29. Holland CJ, MacLachlan BJ, Bianchi V, Hesketh SJ, Morgan R, Vickery $\mathrm{O}$, et al. In silico and structural analyses demonstrate that intrinsic protein motions guide $\mathrm{T}$ cell receptor complementarity determining region loop flexibility. Front Immunol. 2018;9:674.

30. Hennecke J, Wiley D. Structure of a complex of the human alpha/beta T cell receptor (TCR) HA1.7, influenza hemagglutinin peptide, and major histocompatibility complex class II molecule, HLA-DR4 (DRA*0101 and DRB1*0401): insight into TCR cross-restriction and alloreactivity. J Exp Med. 2002;195(5):571-81.

31. Beaulieu ME, Jauset T, Masso-Valles D, Martinez-Martin S, Rahl P, Maltais L, et al. Intrinsic cell-penetrating activity propels Omomyc from proof of concept to viable anti-MYC therapy. Sci Translat Med. 2019;11(484).

32. Luo M. Influenza Virus Entry. In: Rossmann MG, Rao VB, editors. Viral Molecular Machines. Boston: Springer US; 2012. p. 201-21.

33. Wang P, Sidney J, Kim Y, Sette A, Lund O, Nielsen M, et al. Peptide binding predictions for HLA DR, DP and DQ molecules. BMC Bioinf. 2010;11:568-80.

34. Schneidman-Duhovny D, Khuri N, Dong G, Winter M, Shifrut E, Friedman N, et al. Predicting CD4 T-cell epitopes based on antigen cleavage, MHCII presentation, and TCR recognition. PLoS One. 2018;13(11):e0206654.
35. Sorde L, Spindeldreher S, Palmer E, Karle A. Tregitopes and impaired antigen presentation: drivers of the immunomodulatory effects of IVIg? Immun Inflamm Dis. 2017;5:400-15.

36. Lombard-Platlet S, Bertolino P, Deng H, Gerlier D, RabourdinCombe C. Inhibition by chloroquine of the class II major histocompatibility complex-restricted presentation of endogenous antigens varies accoding to the cellular origin of the antigen-presneting cells, and the nature of the T-cell epitope, and the responding cell. Immunology. 1993;80:566-73.

37. Schmitt TM, Aggen DH, Ishida-Tsubota K, Ochsenreither S, Kranz DM, Greenberg PD. Generation of higher affinity T cell receptors by antigen-driven differentiation of progenitor $\mathrm{T}$ cells in vitro. Nat Biotechnol. 2017;35:1188-95.

38. Wagner EK, Qerqez AN, Stevens CA, Nguyen AW, Delidakis G, Maynard JA. Human cytomegalovirus-specific T-cell receptor engineered for high affinity and soluble expression using mammalian cell display. J Biol Chem. 2019;294:5790-804.

39. He Q, Liu Z, Liu Z, Lai Y, Zhou X, Weng J. TCR-like antibodies in cancer immunotherapy. J Hematol Oncol. 2019;12:1-13.

40. Chang AY, Gejman RS, Brea EJ, Oh CY, Mathias MD, Pankov $\mathrm{D}$, et al. Opportunities and challenges for TCR mimic antibodies in cancer therapy. Expert Opin Biol Ther. 2016;16:979-87.

41. Inaguma Y, Akahori Y, Murayama Y, Shiraishi K, Tsuzuki-Iba $\mathrm{S}$, Endoh A, et al. Construction and molecular characterization of a T-cell receptor-like antibody and CAR-T cells specific for minor histocompatibility antigen HA-1H. Gene Ther. 2014;21:575-84.

42. Denkberg G, Lev A, Eisenbach L, Benhar I, Reiter Y. Selective targeting of melanoma and APCs using a recombinant antibody with TCR-like specificity directed toward a melanoma differentiation antigen. J Immunol. 2003;171:2197-207.

43. Rosskopf S, Leitner J, Paster W, Morton LT, Hagedoorn RS, Steinberger P, et al. A Jurkat 76 based triple parameter reporter system to evaluate TCR functions and adoptive T cell strategies. Oncotarget. 2018;9:17608-19.

44. Lund O, Nielsen M, Kesmir C, Petersen AG, Lundegaard C, Worning $\mathrm{P}$, et al. Definition of supertypes for HLA molecules using clustering of specificity matrices. Immunogenetics. 2004;55(12):797-810.

45. Sette A, Vitiello A, Reherman B, Fowler P, Nayersina R, Kast $\mathrm{WM}$, et al. The relationship between class I binding affinity and immunogenicity of potential cytotoxic $\mathrm{T}$ cell epitopes. J Immunol. 1994;153:5586-92.

46. Topalian SL, Rivoltini L, Mancini M, Ng J, Hartzman RJ, Rosenberg SA. Melanoma-specific CD4+ T lymphocytes recognize human melanoma antigens processed and presented by epstein-barr virus-transformed B cells. Int J Cancer. 1994;58:6979.

47. Leung CS, Maurer MA, Meixlsperger S, Lippmann A, Cheong C, Zuo J, et al. Robust T-cell stimulation by Epstein-Barr virustransformed B cells after antigen targeting to DEC-205. Blood. 2013;121:1584-94.

48. Issekutz T, Chu E, Geha RS. Antigen presentation by human B cells: $\mathrm{T}$ cell proliferation induced by Epstein Barr virus B lymphoblastoid cells. J Immunol. 1982;129:1446-50.

49. Purner MB, Berens RL, Krug EC, Curiel TJ. Epstein-Barr virus-transformed B cells, a potentially convenient source of autologous antigen-presenting cells for the propagation of certain human cytotoxic $\mathrm{T}$ lymphocytes. Clin Diagn Lab Immunol. 1994;1:696-700.

50. Chen M, Shirai M, Liu Z, Arichi T, Takahashi H, Nishioka M. Efficient class II major histocompatibility complex presentation of endogenously synthesized hepatitis $C$ virus core protein by EBV transformed B lyphoblastoid cell lines to CD4 T cells. J Virol. 1998;72:8301-8.

Publisher's NoteSpringer Nature remains neutral with regard to jurisdictional claims in published maps and institutional affiliations. 